\title{
Artisans and Digital Craft in Latin America: The contribution of architects to their creativity and production
}

\section{SIGRADI2018 TECHNOPOLITICAS \\ xxii congresso da sociedade iberoamericana de gráfica digital 22th conference of the iberoamerican society of digital graphics $07|08| 09 \mid$ novembro|2018 iau usp | são carlos | sp br}

\author{
Pablo C. Herrera \\ Universidad Peruana de Ciencias Aplicadas | Perú | pablo@espaciosdigitales.org
}

\begin{abstract}
This research explores the work of a generation of Latin-American architects who use programming and fabrication with traditional artisans. In the 21 st century, this scenario was empowered from experiences produced in Fab Labs and Makerspaces in the context of localisms. We look at how digital technologies improve their processes, focusing on creation, adapting to the new economy, strengthening the regional identity in the scene of globalized Design, when political discourse drives innovation and technology to its benefit. The main objective is to understand the coexistence of designers and traditional artisans, providing experiences that could strengthen the identity of design in the region.
\end{abstract}

Keywords: Artisan, Digital Craft, Digital Fabrication, Latin America.

\section{INTRODUCCIÓN}

Treré y Barranquero (2017) evidenciaron que la popularización académica de la tecnopolítica en Latinoamérica apareció a partir de la segunda década del siglo XXI, a pesar que el impacto de la política y tecnología se estudió a lo largo de la historia de los artefactos tecnológicos o Estudios en Ciencia y Tecnología desde hace más de treinta años. Impulsado por movimientos sociales asociados a las redes y medios digitales, su estudio creció con las campañas presidenciales de Barack Obama, Donald Trump o Dilma Rousseff. En ese contexto, tecnologías como la fabricación digital no son una excepción como el "mass making" tampoco lo fue para el discurso político en China. Bajo ese amparo tecnopolítico y considerando que el término Fab Lab tiene el alcance de un movimiento social (Walter-Herrmann, 2016, p.38), Lindtner y Avle (2017) evidenciaron como "los líderes políticos aprovecharon las promesas de la fabricación digital, presionando a los ciudadanos a volverse auto-empresarios y colectivamente innovadores" (p.12). Así como sucede con la Tecnocracia en donde equipos técnicos lideran o marcan la línea de un gobierno, Lindtner y Avle también sostienen que los políticos se vinculan a las tecnologías para proyectar su liderazgo global, impulsar redes tecno científicas y mitigar algunos problemas como el empleo. (p. 1). Ese discurso político, que impulsó iniciativas asociadas a la fabricación digital en el hemisferio norte, no tuvo el mismo impacto en Latinoamérica. Entre el 2015 y el 2018 el crecimiento de los Fab Labs en América del Sur se mantuvo anualmente en alrededor del $8 \%$, mientras que el crecimiento mundial en el mismo periodo fue de $134 \%$.

Lombana (2018, p.359) sostiene que en Latinoamérica se intenta cerrar brechas a diferentes carencias y de acceso al conocimiento y conectividad con iniciativas como la "red de clubes maker en colegios públicos de Chile, el centro de innovación comunitaria C-Innova en Colombia
(Reynolds-Cuéllar, 2018), los campamentos creativos Maker, Tinker, Learn en Centroamérica (Samson et al., 2018), el programa Conectar Igualdad en Argentina y el concurso Technovation en Bolivia (Velásquez, 2018)". Por diferentes circunstancias (principalmente económicas y de gestión), la fabricación digital en Latinoamérica se diluye en una ilusión e incertidumbre sobre su sostenibilidad, como sucedió con el proyecto One Laptop Per Child en Latinoamérica (Cristia et al., 2012). Todo esto evolucionó y finalizó por la misma naturaleza de su orígen (Herrera y Juárez, 2013) así como por su replicación criticada por el Politics of Fabrication Laboratory desde el 2011. Por lo tanto, buscar soluciones de implementación y vigencia sigue siendo un problema a alcanzar que enfrentan otros continentes como África (Mboa, 2017).

Pero en este momento, ¿Quiénes y cómo contribuyen a sostener promesas políticas, impulsando la identidad y cultura de un país, beneficiando al mismo tiempo a quienes las impulsan? ¿Cuáles son esas experiencias que nos llevarían fortalecer un discurso político impulsado por la tecnología en Latinoamérica?

En esta investigación se encontró una contribución significativa desde la experiencia del arquitecto en el quehacer artesanal, en momentos en que el discurso de los gobiernos impulsan la innovación y tecnología para su beneficio. Así se analizó casos que incluyen distintas maneras de acercarse a comunidades de artesanos, analizando los orígenes de su implementación, que incluye el uso de código para impulsar la creatividad y procesos en vez de sólo resultados a través de la fabricación digital.

Así mismo, la propuesta de esta investigación coincide con la búsqueda de algunas universidades de la región con discursos tecnopolíticos que desean fortalecer la 
educación del arquitecto, cuando las técnicas digitales no son una opción, sino una tendencia. Decidir por una implementación computarizada (usuarios de software) o computacional (programadores de software) es un tema aún indefinido en muchas escuelas de arquitectura, en donde la academia asume una posición política para integrarlas de manera empírica o desde propuestas autogestionarias de sus profesores, tanto como el gobierno desea hacerlas suyas. Los métodos utilizados por y para los artesanos podrían servir de contexto para futuras implementaciones educativas.

Por lo tanto, es necesario definir líneas de acción dentro de esta coyuntura, que no sólo se encaminen en el contexto académico de la arquitectura, sino de la sociedad.

\section{¿TECNOPOLÍTICA?}

Una serie de hechos al finalizar la primera década del siglo XXI, concilian el tema de este congreso junto a la contribución computacional y de fabricación de la arquitectura en la creatividad y producción artesanal. En el 2009, Gabriele Heecht sociabiliza el término tecnopolítica "para referirse a la práctica estratégica de diseñar o usar tecnología para constituir, incorporar o promulgar objetivos políticos" (p.15). Ese mismo año, expiró la patente Fused Depositing Modeling (FDM) y nació también Makerbot Industries que usa la misma tecnología de extrusión de filamento. Por su costo y accesibilidad, abrió enormes posibilidades en la fabricación digital, considerando que esta es la principal infraestructura en las facultades de arquitectura latinoamericanas (Sperling, Herrera y Scheeren, 2015).

También en el 2009, la Universidad Nacional de Ingeniería en Perú y la Addis-Ababa University en Etiopía se convirtieron en las dos primeras sedes del proyecto FAB Academy, una iniciativa mundial con 660 graduados al 2017 (13\% de Latinoamérica) que hasta el 2018 especializó e impulsó la creación de 1284 FabLabs, de los cuales un 10\% representa a nuestra región. Gershenfeld (2005) sostiene que las tecnologías en los Fab Labs se crearon para impulsar la producción local a través de la solución a sus propios problemas y como se encontró en esta investigación, la producción local fue uno de los temas que los alumnos utilizaron en sus proyectos. Troxler (2010) propuso a los Fab Labs como modelo de negocio mientras provean infraestructura e innovación y esa característica ha sido fundamental en la academia y profesión que creó sinergia con los artesanos. Con este modelo, el avance de la fabricación digital superó la afirmación de Bonsiepe (1985), y se demostró que a través de las actuales tecnologías no se requiere de una política-económica para que un pueblo desarrolle sus diseños, ni esperanza política que democratice los medios para producirlos, porque ello sucede desde la segunda década del siglo XXI, pero si de pensar en una política que la haga sostenible.

\section{ARTESANOS Y TECNOLOGÍA}

En Latinoamérica, la integración de tecnologías en las habilidades del diseñador y artesano fue estudiada por Duque et al. (2005, p. 73) al analizar métodos computarizados que incentivan la creatividad, la visualización y su contextualización. Sin embargo, esta interacción no demostró una diferencia en la producción física hasta la aparición de los laboratorios de fabricación digital que facilitaron a los diseñadores, herramientas que superaron la representación del objeto, por otras como la preservación de los procesos a través del código, así como la fabricación y adaptación de máquinas. En ese sentido, los casos de estudio presentados en esta investigación, con sus instalaciones móviles e instalaciones en centros académicos aportan al fortalecimiento de la identidad local, con agenda en la artesanía y de la comunidad.

La artesanía y la fabricación digital se documentaron en una investigación previa con énfasis en la mejora de la producción (Herrera, 2016). En esa misma investigación se resaltó iniciativas en América del Sur con escasas evidencias del norte de la región como sucede ahora. También se evidenció que el total de los arquitectos usan programación visual (Grasshopper) y no escrita, dependiendo del nivel de complejidad de los diseños, apoyando indirectamente a la creatividad artesanal. Ese panorama en los dos últimos años ha cambiado como se presenta en este estudio, considerando que la perspectiva computacional en la artesanía tiene un precedente importante en los trabajos de Kornhauser (2002) y Jacobs (2013) de MIT, que crearon entornos programados para sistematizar procesos creativos con el fin de potenciar la artesanía.

\section{¿POR QUÉ LA ARTESANÍA?}

Aish (2005) sostiene que "el diseño es diferente a la artesanía (porque el diseñador no actúa directamente sobre el material, pero tiene una forma más indirecta, y posiblemente más poderosa de controlar la materialización)." (p.10). en ese contexto, Aish (2005) propuso que "el advenimiento de la fabricación controlada digitalmente significa que el diseñador "con conciencia geométrica" y "capacitado computacionalmente" está tan cerca de la materialización como en el proceso artesanal original, pero con precisión y control con la capacidad de explorar la variación que antes era inimaginable."

Esa diferencia, se vuelve una ventaja en ambos sentidos cuando la artesanía aporta al aprendizaje del diseño y el diseño aporta al proceso creativo y productivo en la artesanía.

Bonsiepe (1985) sostiene que "en los productos artesanales el proceso proyectual y la fase de producción están operativamente unidos en la misma persona, mientras que en los productos industriales ambos procesos están funcionalmente diferenciados" (p.64) como sucede incluso en la arquitectura. Oxman (2007) sostuvo que efectivamente ello se evidencia por la profesión de herramientas de fabricación digital, pero al mismo tiempo sugiere "que ciertas habilidades adquiridas a través de procesos de personalización pueden contribuir a los protocolos de diseño guiados por fabricación." (p.1). Neri Oxman denominó a esta sinergia Digital Craft [Artesanía Digital].

\section{ARTESANÍA DIGITAL [DIGITAL CRAFT] Y ARQUITECTURA}

Bonsiepe (1985) propuso una manera de acercarse al pueblo (artesanos) antes que la fabricación digital sea 
accesible, a través de "la participación del profesional como catalizador." (p. 65). La propuesta de esta investigación devela que tanto la programación y fabricación digital en manos de profesionales como los arquitectos, promueve y preserva no sólo las tradiciones de un país, representadas por la artesanía, sino también de la pedagogía y enseñanza de la arquitectura, a través del enriquecimiento de la habilidad artesanal de la sociedad y del pensamiento computacional en los estudiantes de arquitectura y diseño a través del concepto Digital Craft [artesanía digital].

El aporte de los arquitectos en la integración de herramientas de fabricación digital y uso de programación visual en el contexto del emprendimiento social en comunidades vulnerables fue presentado en SIGraDi (Bernardo et al., 2014; Júdice et al., 2016), bajo el mismo interés de Noel (2016), quien sostiene que los académicos han estado mirando el potencial de la artesanía como herramienta de enseñanza. Por otro lado, creemos que uno de los aportes más importantes para el artesano es el de mejorar las técnicas artesanales cuando se codifican sus procesos, como parte del proceso del Digital Craft.

Pero Senske (2014) sostiene que "Digital Craft es una noción todavía en evolución en la arquitectura" (p.841) y afirma que "los arquitectos entienden aún menos sobre la artesanía digital como un proceso". Sin embargo, a partir del trabajo de Wing (2006), Senske (2014) sostiene que el pensamiento computacional es esencial para definir y producir un buen Digital Craft. Al modo de decir de Kudless (2016), "Digital Craft no es un estilo, sino una actitud de diseño que maximiza el poder de la computación digital para coordinar la complejidad, integrar el conocimiento y fabricar arquitecturas avanzadas." (p.17). En diez años, la fabricación digital se documentó en Europa y EE.UU. dentro del concepto de artesanía, como una práctica con tradición y origen que difiere del proceso artesanal existente en Latinoamérica, por ello se propone explorar aún más esa diferencia entre el norte y el sur.

\section{HEMISFERIOS NORTE Y SUR}

A diferencia del norte, Los laboratorios de fabricación digital en Latinoamérica se enfrentan a cuatro factores adversos: Económico; Gestión y Mantenimiento; Administrativo y Educativo (Herrera y Juárez, 2013). Factores que también considera Mboa (2017, p. 82-83) al analizar el caso de la africanización del movimiento maker.

A ello se añaden cuatro escenarios desde donde se desarrolla intensamente la actividad artesanal: 1) según Canclini (2004, p.34) son el resultado de habilidades populares indígenas y campesinas; 2) según Lauer (1982 pp. 64-65) desde una tradición andina con base en culturas pre coloniales como la Inca y Maya; 3) según Borges (2015, p.11) desde núcleos humanos de la periferia, zonas rurales, favelas y desde áreas marginales a las ciudades como efecto de la migración del campo a la ciudad, produciendo objetos de manera colectiva; y 4) según Sabogal (1974) y Rengifo (1989) su desarrollo se origina desde una demanda del mercado que toma la primera generación de productos como referencia para producir nuevas versiones. En el siglo XXI este escenario asociado a los factores adversos se refuerzan como resultado de una producción de experiencias en Fab Labs, Makerspaces y laboratorios en el contexto de la neo artesanía (Autor). En esta investigación se discuten nuevos escenarios, como resultado del análisis de nuevos casos de estudio.

Lindtner y Avle (2017) sostienen que, las economías del sur son similares a los ideales del código abierto porque son informales, de imitación, artesanales. Nosotros coincidimos en que la principal diferencia entre el norte y el sur, es el nivel de educación de su audiencia. Para Borges (2011) en el norte "las técnicas se aprenden en cursos universitarios y son prácticadas por personas educadas que ven en esta actividad su autoexpresión, y esto los acerca más al arte que al diseño". (p. 25). Esta situación no es diferente en los Fab Labs, porque su audiencia es mucho más parecida a la del norte que a la realidad del sur, es decir, encontramos profesionales entrenados en estos espacios. Waldman-Brown et al. (2013) encontraron que en los Fab Lab de África la audiencia son "público de académicos y aficionados de clase alta: bien educados pero imprácticos" en oposición a aquellos que realizan manufactura: "una audiencia de artesanos informales de clase baja: sin educación, crudo y peligrosos" (p.18). Los temas en el norte, como lo sostienen Seo-Zindy and Heeks (2017) tienden a orientarse a problemas sociales como la sostenibilidad ambiental (p.13), al contrario, los del sur enfrentan otras problemáticas más cercanas a su propia realidad.

Para el sur, Borges (2011) sostiene que los objetos son "hechos a menudo colectivamente (por familia y / o grupos de vecinos) (...). Muchas de estas técnicas se han transmitido a través de generaciones de la misma familia o por uno o más miembros mayores de una comunidad (...). Rara vez se han aprendido en la escuela, incluso cuando los grupos artesanales pertenecen a la clase media." (p. 25).

A pesar que ambos hemisferios demuestran similitudes en algunas de sus características, la economía es quizás el factor más importante en su diferenciación. Morel et al. (2015) argumentan que "estos espacios (Fab Labs) están haciendo un buen trabajo en sus funciones de acelerar la innovación colaborativa, (...) sirviendo a un tipo privilegiado de usuarios y muy a menudo no a las PYME" (p. 1). El apoyo de los Fab Labs ha ido impulsando a las Pequeñas y Medianas Empresas (PYMEs) artesanales, como el caso de la Université de Lorraine en Francia y su primer Mobile Fab Lab France en el 2014. Las PYME son una de las bases artesanales más importantes en Europa y para el modelo de los Fab Lab son la principal clientela junto a los innovadores e investigadores (Troxler \& Wolf, 2010). Sin embargo, en Latinoamérica con una economía muy variable, la Micro Empresa e incluso la informal es lo más común y en ese grupo se incluyen migrantes y poblaciones de escasos recursos que en el gran porcentaje de los casos analizados está presente.

\section{METODOLOGÍA}

Para la selección de casos se revisaron dos bases de datos asociadas a tecnologías digitales en dos contextos académicos: publicaciones en conferencias arbitradas (CumlnCAD) y desarrollo práctico (FAB Academy). Además, se incluyeron profesionales a través de la
3 
revisión de exhibiciones sobre fabricación digital. Se hizo una búsqueda a nivel de palabras clave en la totalidad de artículos de CumlnCAD (http://papers.cumincad.org/). Se encontró que la producción científica para la búsqueda de casos de estudio por palabras clave dio un total de 52 artículos. En idioma español las palabras clave artesan* [artesanal (1), artesanales (1), artesanía (1)] alcanzó 3 artículos. En idioma inglés, las palabras clave craft* [craft (26), crafting (10), craftingnumerical (1), crafts (4), craftsman (1), craftmanship (4) y craftpersona (1)] sumaron 47 artículos. A ellos se sumaron 2 artículos con las palabras clave: artisanship (1) y artisan (1). Se procedió a lectura del total de artículos a partir de la metodología PRISMA (Preferred Reporting of Items for Systematic Reviews and Meta-Analysis) utilizada por Seo-Zindy \& Heeks (2017) en su investigación sobre Makerspaces y Fab Labs en el hemisferio sur. A partir de esa filtración se seleccionó los casos de estudio relevantes para las preguntas del estudio.

Al revisar los Class Archive del FAB Academy (20082017) con el fin de buscar iniciativas, se analizaron 86 trabajos del FAB Academy que representan el 13\% del total mundial producido sólo en Latinoamérica. Fueron cuatro trabajos entre el 2012 y el 2014 vinculados al trabajo formal e informal con artesanos y que fueron desarrollados por arquitectos con proyectos de Walter Gonzáles (telares) y Diego Machuca (Vivienda cinética interactiva) en el Fab Academy 2012; de Gonzalo Pérez (máquina para telares) en el Fab Academy 2013 y de Vaneza Caycho (mobiliario) en el Fab Academy 2014. Aunque el trabajo de las arquitectas Roxana Garrido (Fab Academy 2012) y Vanessa Montezuma (Fab Academy 2013) no se asoció directamente con artesanos, su experiencia en el programa los vinculó con ellos para el desarrollo de objetos como el mate burilado y la carpintería después. En su conjunto, fueron parte del proceso de aprendizaje del FAB Academy y su alcance fue específico al aprendizaje.

Finalmente, se analizaron a los participantes latinoamericanos en cuatro exhibiciones organizadas por The Museum of Arts and Design (New Territories: Laboratories for Design, Craft and Art in Latin America, 2014); CAAD Futures (Homo Faber: Digital Fabrication in Latin America, 2015) y SIGGRAPH (Hybrid Craft, 2015 y Unsettled Artifacts: Technological Speculations from Latin America, 2017) que forman parte principalmente de las iniciativas profesionales.

\section{CASOS DE ESTUDIO}

En un estudio previo acerca de la experiencia en programación y fabricación digital entre artesanos y arquitectos (Herrera, 2016) se identificó cuatro grupos experiencias Latinoamericanas: Formativas; Académicas; Prácticas y Neo artesanales. En este artículo se sintetiza la categorización a partir del contexto en donde se gestó la propuesta.

Del universo de experiencias analizadas, se presentan aquellas que representan aportes importantes a los objetivos del estudio. Agrupando las iniciativas en tres categorías: Académicas, Profesionales y Artesanales con el fin de dejar abierta la posibilidad a estudios específicos.

\section{INICIATIVAS ACADÉMICAS}

Las universidades estatales y privadas de Latinoamérica reúnen la mayor cantidad de laboratorios de fabricación digital, utilizando fondos nacionales concursables o de sus propias universidades como sucedió en MIT (EE.UU.) a través de la National Science Foundation. En esta categoría se agrupan experiencias que buscan alfabetizar tecnológicamente y acercarse a una comunidad saliendo de sus instalaciones y llevando su infraestructura al contexto. Estos se conocen como laboratorios móviles, que llegan a diferentes audiencias, especialmente en la periferia, fuera de las grandes ciudades. Entre el 2001 y 2004 el Learning Independence Networks (LIN) o Esperanza de MIT potenció la tecnología de los países por sobre la importación de las mismas, y permitió al Instituto Tecnológico de Costa Rica en Cártago desarrollar el proyecto "Aprender Independencia" a cargo de Bakhtiar Mikhak. Su iniciativa se presentó en el FAB1 (2005) bajo el concepto de Fab Labs Camps que incluía un Mobile Fab Lab que visitó diferentes escuelas de la región. En el 2007, MIT desarrolló su primer Mobile Fab Lab, construyendo entre el 2009 y 2015 cuatro más en sociedad con municipalidades. Este modelo de implementación se ha replicado para visitar comunidades artesanales a través de tres iniciativas en Latinoamérica.

En Latinoamérica, esta aproximación tiene origen en las escuelas de arquitectura. En Chile, a través del Fondo de Innovación para la Competitividad (FIC) del Gobierno Regional de Valparaíso se financió en el 2014 el Aconcagua Fab Lab (móvil) por la Escuela de Arquitectura y Diseño de la Pontificia Universidad Católica de Valparaíso. De acuerdo a la PUCV, con la convocatoria a talleres abiertos (en espacios abiertos como plazas) y otros especializados el proyecto se orientó a micro y pequeños artesanos con experiencia. En el 2017 inician el proyecto Móvil Maker para realizar experiencias en Atacama, al norte de Chile. En México la Universidad UMED implementó el Fab Lab Monterrey Mobil el 2017 a cargo de la arquitecta Daniela Frogheri. En Costa Rica, se creó el Fab Lab Movil Kolbi Veritas de la Universidad Veritas de Costa Rica (2017) bajo la dirección del arquitecto Robert Garita. En Brasil, la Kombi Pronto 3D (móvil) que empezó el 2018 por una iniciativa del laboratorio del mismo nombre en la Universidade Federal de Santa Catarina en el 2013, impulsa la sociabilización de técnicas de fabricación en escuelas y comunidades.

Este emprendimiento se formalizó en el FAB13 (2017) y se creó el Mobile LaT Labs Network impulsado por Robert Garita de Costa Rica. Iniciativas como el Floating Fab Lab Amazon presentado en el FAB9 (2013) por Benito Juárez han tenido el mismo alcance, acercarse a través de la tecnología a potenciar el trabajo manual de la población rural.

\section{INICIATIVAS PROFESIONALES}

Desde el trabajo de los argentinos Paula Gaetano (artista) y Gustavo Crembil (arquitecto) y su proyecto Mestizo Robotics (iniciado en la villa San Roque de la amazonía peruana) hasta las Exhibiciones realizadas en los EE.UU. se resalta la integración de profesionales y habilidades en tecnología uniendo esfuerzos con artesanos y comunidades en Latinoamérica que demuestran un común denominador colaborativo. Esta selección de 
profesionales tiene diferentes contextos demostrado en exhibiciones como New Territories: Laboratories for Design, Craft and Art In Latin America (Stokes, 2014) que reunió el trabajo de gt2P (Chile) y del Studio Guto Requena (Brasil), demostrando como el trabajo con comunidades artesanas ha producido ejemplo de objetos hechos a mano bajo una producción conceptualizada como Digital Crafting. Homo Faber: Digital Fabrication in Latin America demostró que las migraciones hacia Latinoamérica de diferentes actores impulsó también estas iniciativas y fueron la base para la actual generación de arquitectos (Sperling y Herrera, 2015), así como las iniciativas artísticas curadas en SIGGRAPH, que por primera vez se enfocan en artistas latinoamericanos trabajando con tecnologías digitales (Fernández, 2017, p.414).

En Perú, Ricardo Torres (Fab Academy 2014) a través de su PYME Lima Makers, desarrolló la producción neo artesana de la empresa de publicidad Origen Peregrino, un estudio de diseño peruano que une el trabajo de la agencia TAG Estudio Gráfico y el diseñador industrial Lau Toyosato para rediseñar piezas artesanales de origen andino reemplazando materiales y diseño con el fin de convertirlo en un producto artístico y comercial. Mate.Bits, también de Perú, es un proyecto impulsado por la arquitecta Roxana Garrido (graduado Fab Academy 2012), que se propuso unificar técnicas de mate burilado y otras de fabricación digital. El Fab Lab UNI participó a través del servicio de máquinas. Todos estos casos han recibido reconocimientos, muchos de ellos alrededor del mundo, desde exhibiciones y premios. Pero una carencia ha sido la falta de documentación de la implementación por ellos mismos.

En Trinidad y Tobago, Noel (2016) directora de VAAN Design Lab impulsó la recuperación de prácticas tradicionales artesanales a través de su reinterpretación con el fin de integrar la computación y tecnología digital. Al contrario de los casos anteriores, estos experimentos fueron definidos bajo tres técnicas de implementación: Digital Crafting (a partir de métodos computacionales se reinterpreta el objetos artesanal para hacer un nuevo artefacto), Computational Crafting (en donde todo el proceso de diseño y fabricación son manuales pero tienen su origen en un método computacional como el shape grammar) y Crafting Fabrication (combina herramientas digitales y no digitales, procesos $y$ tecnologías). Está a nuestro entender, es la que mejor avanza hacia una metodología de implementación en los procesos de aprendizaje del diseño.

\section{INICIATIVAS ARTESANALES}

Estos casos son iniciativas que obtuvieron fondos públicos o privados para el proyecto, utilizando la infraestructura y personal de un Fab Lab, tanto para la capacitación como para la producción de los objetos. Ese fue el caso dirigido por Vanessa Roca en Perú, un proyecto PYME en donde un grupo de joyeros buscaron capacitación en el Fab Lab UNI que es administrado por la Facultad de Arquitectura, Urbanismo y Artes de la Universidad Nacional de Ingeniería, para explorar nuevas tecnologías aplicadas a sus procesos, como la impresión 3D (stereolitografía) para la orfebrería artesanal.
En un contexto regional el Proyecto Fab Craft: Artesanias Digitales es una plataforma autogestionaria que forma parte de la Red de Fab Labs de Latinoamérica y es coordinada por la arquitecta Trinidad Gómez, junto a arquitectos y diseñadores de Bolivia, Costa Rica, Ecuador, México y Perú desde el año 2016. Sus miembros, vieron en la tecnología de fabricación digital un nexo en talleres realizados en sus países. En el año 2017 anunciaron su Manifiesto Fab Craft en el FAB13 (Santiago de Chile), con un objetivo muy claro: no pretender reemplazar a los artesanos por máquinas, sino darles la facilidad para utilizarlas.

\section{RESULTADOS}

Aunque es conocida la posición de Sennett (2008) con respecto al artesano, la producción artesanal tradicional Latinoamericana se desarrolla con una habilidad y conocimiento que no está exteriorizada o expuesta de manera explícita, sino encerrada en sus autores evolucionando por ensayo y error entre generaciones. En palabras de Bonsiepe (1985) "una capacidad proyectual nada despreciable, informal, fuera de los circuitos de la formación oficial" (p. 249). Bonsiepe (2012) lo define como "un know-how comprendido como tipo de saber que no puede ser codificado y no puede ser adquirido mediante métodos discursivos" (p. 87).

Pero, se encontró en los casos de estudio que la programación escrita (Noel, 2016) y visual (gt2p y Requena), demostraron que facilita codificar procesos y por lo tanto la posibilidad de almacenar un conocimiento como se analizó en esta investigación, impulsando el trabajo entre el diseñador y el artesano.

Aunque los espacios que albergan estas iniciativas pueden ser las universidades, es evidente que el movimiento Fab Lab es el que enmarca el contexto. En investigaciones previas sobre fabricación digital en la región, las iniciativas se impulsaron por movimientos migratorios de arquitectos latinoamericanos que retornaron a sus países de origen después de estudiar una maestría o doctorado (Herrera, 2011). En esta investigación, encontramos que estas iniciativas fueron llevadas adelante por una generación de nuevos arquitectos nacidos en la década de 1980 con estudios en sus propios países especialmente dentro del Fab Academy, lo que demuestra el importante efecto de la generación anterior que dió las bases, especialmente en la infraestructura y equipos.

Algunos establecieron sinergias y otros sólo tomaron la artesanía como referencia de nuevos diseños. Las herramientas utilizadas en la mayoría de casos se creó o adaptó reemplazando equipos convencionales de fabricación, concluyendo que la relación "arquitecto y artesano" es coincidente con la tendencia de "maquinas que hacen máquinas" propuesta por Lassiter (2013, p. 253) con el fin mejorar el objeto original, como respuesta a otras tecnologías que simplemente se centraron en la visualización. Estos resultados inciden directamente en el valor de la artesanía, que pueden mejor la calidad de vida y dar más tiempo al artesano para crear, con el fin de replicar en otros contextos a través de laboratorios móviles.
5 
Por otro lado, los diseñadores industriales se reapropiaron de objetos tradicionales como referencias para establecer nuevas líneas de diseño, lo que abre una nueva línea de producción independiente.

Consideramos que los resultados alcanzados revelan que la programación y fabricación ha sido relevante para los artesanos y ha significado un aprendizaje de procesos para arquitectos y diseñadores. Sin embargo, es claro que la sistematización de los resultados de cada propuesta ha sido más sostenible en cuento mayor investigación sistematizada se ha hecho. Si bien es cierto, los casos de estudio demuestran reconocimiento en exhibiciones y concursos, la falta de documentación sobre sus procesos de implementación evoluciona entre el ensayo y el error. A diferencia de las propuestas de casos de estudio desde los EE.UU. por ejemplo, que conducen finalmente a la creación de políticas de fabricación, como las que Latinoamérica no produce y han criticado Alvarez y Gonzáles (2016) desde que propusieron sus talleres en los Visiting School el 2011. Ello, es una urgente necesidad que superar. De otra manera, a pesar que el código potenció y preservó procesos, los mismos casos de estudio carentes de una investigación más exhaustiva, sólo se quedarán como una propuesta disruptiva en la historia del diseño latinoamericano. En el hemisferio norte, se impulsó la cultura maker y ahora la crafter con incidencia en la educación e incluso del diseño, retomando los ideales de movimiento británico Arts and Crafts de Ruskin y Morris (Garber, Hochtritt \& Sharma, 2018)

Este punto es importante porque esta sinergia tanto para artesanos y arquitectos, desencadenó un incentivo que los llevó a pensar más en el proceso que sólo en el objeto. Analizando el proceso de otros y repensarlo para producir código abrió posibilidades para potenciar otros diseños. La introducción de la fabricación permitió superar la simple representación a la que estaban acostumbrados los artesanos tradicionales, viendo en el resultado una posibilidad para reinventar sus propuestas.

\section{DISCUSIÓN}

La investigación del saber hacer del artesano y la personalización del proceso de diseño alcanzado por artesanos latinoamericanos en colaboración con diseñadores especialistas en técnicas de programación y fabricación digital, permitirá un mayor acercamiento al pensamiento computacional potenciando el proceso sobre el resultado. Aprendiendo procesos de diseño en el que se consideran diversos materiales (madera, cuero, arcilla, bronce, metal, porcelana, etc.), fabricación y ensambles interactuando a escala objetual bajo un entorno computacional, evolucionaremos hacia experiencias de escala arquitectónica. Esta adaptación gradual es sostenible, por cuanto nuestras economías no permiten inversiones educativas para experimentar de manera directa con prototipos a escala arquitectónica. Por ello, acercarse a la artesanía, es una oportunidad de entender procesos de manera formativa. Esta investigación aporta experiencias que fortalecen la identidad del diseño contemporáneo en la región, proponiendo insumos de experimentación a personas que dirigen programas educativos, laboratorios de fabricación, talleres y oficinas de gobierno, con el fin de proponerles establecer una sinergia entre las tradiciones populares y profesionales de distintas disciplinas en una interacción académica con la sociedad a través de políticas sostenibles.

Como puntualizó Montaner y Muxí (2011), "la acción política desde la arquitectura siempre ha existido, a pesar de que hay profesionales que niegan tal relación y que hacen política por omisión. Si la política es la organización social de un grupo que se desarrolla en un espacio, según desde donde se actúe en la creación de este espacio, este será integrador o segregador, inclusivo o exclusivo, se regirá según la aspiración a la redistribución a la calidad de vida o según la perpetuación de la exclusión y del dominio de los poderes. Es por ello que la arquitectura es siempre política." (pp. 65-66). La perspectiva de esta investigación, propone una aproximación para discusiones a muy corto plazo que impulsarán tanto la artesanía como la educación de los arquitectos próximos a iniciar la tercera década del siglo XXI. Como anticipan Valsiner, Lutsenko y Antoniouk: "La tarea de preparación de los knowledge makers necesita ser resuelta por personas que realmente guían la Bildung, en lugar de políticos de horizontes temporales limitados en sus carreras o contadores para quienes economizar inmediatamente en sus actuales instalaciones borra un horizonte de futuras ganancias". (2018, p.ix), ese es finalmente nuestro reto.

\section{AGRADECIMENTOS}

A la Dirección de Investigación de la Universidad Peruana de Ciencias Aplicadas y a cada uno de los arquitectos que participaron en diferentes encuestas para esta investigación, compartiendo su historia.

\section{REFERENCIAS}

Alvarez, N. \& Gonzaléz, F. (2016). Política y Fabricación Digital. Una discusión en curso. Sevilla: Vivok Works.

Bernardo, M. \& Cabral, J. (2014). Fabricação digital e variedade fora do contexto industrial. (pp. 320-323). In Proceedings of the XVIII Conference of the Iberoamerican Society of Digital Graphics: Design in Freedom. São Paulo: Blucher.

Bonsiepe, G. (1985). El diseño de la periferia: Debates y Experiencias. Barcelona: Editorial Gustavo Gilli.

Bonsiepe, G. (2012). Design: como prática de projeto. Sao Paulo: Blucher.

Borges, A. (2011). Design + Craft. The Brazilian Path. Sao Paulo: Editora Terceiro Nome.

Borges, A. (2013). Craft revitalization as a change agent in Latin America. Making Futures Journal 3, pp. 11-14. Retrieved from

http://www.plymouthart.ac.uk/documents/Adelia_Borges_Keynote.pdf

Cristia, J., Ibarrán, P., Cueto, S. \& Severín, E. (2012). Tecnología y desarrollo en la niñez: Evidencia del programa Una Laptop por Niño. Washington: Banco Interamericano de Desarrollo. Retrieved from https://publications.iadb.org/bitstream/handle/11319/3919/Te cnolog\%C3\%ADa\%20y\%20desarrollo\%20infantil\%3A\%20Evi dencia\%20del\%20programa\%20\%60Una\%20computadora\% 20por\%20ni\%C3\%B10\%60.pdf?sequence=4.

Duque, C., Sethi, R. \& Vencatachellum, I. (2005). Designers meet Artisans. Nueva Dehli: Craft Revival Trust.

Fernandez, M. (2017). Antecedentes. Leonardo 50(4) 414-417. 
Garber, E., Hochtritt, L. \& Sharma, M. (2018). Makers, Crafters, Educators. Working for Cultural Change. New York: Routledge.

García, N. (2004). Diferentes, desiguales y desconectados. Mapas de la interculturalidad. Barcelona: Editorial Gedisa.

Gershenfeld, N. (2005). Fab: The Coming Revolution on Your Desktop--from Personal Computers to Personal Fabrication. New York: Basic Books.

Henriques, GC. (2009). Crafting New Artefacts. Expressing the changing condition of nature, culture and technology. In Proceedings of the 14th CAADRIA Conference, Dunlin. Retrieved from http://papers.cumincad.org/data/works/att/caadria2009_152.c ontent.pdf

Herrera, P. C. (2016). Digital fabrication and revival craft in Latin America: Alliance between designers and artisans. In: Wong, W., Kikuchi, Y. \& Lin, T. (Eds.). Making Trans/National Contemporary Design History. ICDHS 2016 - 10th Conference of the International Committee for Design History \& Design Studies. São Paulo: Blucher, 2016. DOI: 10.5151/despro-icdhs2016-03_019.

Herrera, P.C. \& Juarez, B. (2013) Fabrication Laboratories: Problems and possibilities of implementation in Latin America, Fab9 Research. Yokohama. http://hdl.handle.net/10757/605215

Herrera, P. C., Montezuma, V., \& Juárez, B. (2018). Crafts in Latin America: The contribution of the Fab Labs in the promotion of resilient communities. In Proceedings from the Fab14 + Fabricating Resilience Research Papers Stream (pp. 57-64). Rotterdam: Rotterdam University of Applied Sciences. http://doi.org/10.5281/zenodo.1344443

Jacobs, J. (2013). Algorithmic Craft: the Synthesis of computational Design, Digital Fabrication, and Hand Craft. MIT MSc in Media Arts and Sciences thesis.

Júdice, A. Maynardes, A., Júdice, M., Aviani, F. (2016). Fabricação digital e identidade de territórios em produtos cotidianos. (pp. 381-386). In XX Congreso de la Sociedad Iberoamericana de Gráfica Digital. São Paulo: Blucher.

Kornhauser, D. (2002). Designing a Craft Computing Environment for Non-Industrial Settings. MIT MSc in Media Arts and Sciences thesis.

Kudless, A. (2016). A Digital Craft Framework. In J. Johnson \& J. Vermillion (Eds.), Digital Design Exercises for Architecture Students (pp. 5-17). New York, NY: Routledge.

Lassiter, S. (2013). FabLabs: Thoughts and Remembrances. In J. Walter-Herrman \& C. Büching (Eds.), FabLabs of Machines, Makers and Inventors (pp. 249-257). Wetzlar: Majuskel Medienproduktion $\mathrm{GmbH}$.

Lindtner, S. \& Avle, S. (2017). Tinkering with Governance: Technopolitics and the Economization of Citizenship. Proceedings of the ACM on Human-Computer Interaction, Volume 1, Issue CSCW, Article No. 70. Retrieved from https://doi.org/10.1145/3134705

Lombana, A. (2018). Economía digital, cultura maker y nuevas formas de creación de valor. In C. Cobo et al. (Eds.), Jóvenes, transformación digital y formas de inclusión en América Latina. (pp. 355-361). Montevideo: Penguin Random House. Retrieved from https://digital.fundacionceibal.edu.uy/jspui/bitstream/1234567 89/229/1/Libro\%20colectivo\%20J\%F3venes\%20Digital.pdf

Mboa, T. (2017). Benefits and the hidden face of the maker movement: Thoughts on its appropriation in African context. LIINC 13(1), pp. 72-88. Retrieved from https://doi.org/10.18617/liinc.v13i1.3774

Montaner, J. \& Muxí, Z. (2011). Arquitectura y Política. Ensayos para mundos alternativos. Barcelona: Editorial Gustavo Gili.

Noel, V. (2016). Crafting as Inquiry into Computation - Exploring wire-bending in traditional practice and design education. (pp.
311-320). In Complexity \& Simplicity - Proceedings of the 34th eCAADe Conference. Finland, August 22-26. Retrieved from

http://papers.cumincad.org/data/works/att/ecaade2016_075.p df

Oxman, N. (2007). Digital Craft: Fabrication Based Design in the Age of Digital Production. In Workshop Proceedings for Ubicomp 2007: International Conference on Ubiquitous Computing. (pp. 534-538). September; Innsbruck, Austria.

Rengifo, A. (1989). La artesanía en la obra de José Sabogal Wiesse. Lima: Centro de Proyectos Integrales.

Reynolds-Cuellar, P. (2018). Artesanos, makers y centros de innovación en Colombia. In C. Cobo et al. (Eds.), Jóvenes, transformación digital y formas de inclusión en América Latina. (pp. 355-361). Montevideo: Penguin Random House

Sabogal, J. (13 de Noviembre de 1974). Artesano y Artesanías. Diario El Comercio.

Samson, K., Velis, E. \& Valladares, C. (2018). Experiencias de cultura maker y educación informal de Mesoamérica. In C. Cobo et al. (Eds.), Jóvenes, transformación digital y formas de inclusión en América Latina. (pp. 377-387). Montevideo: Penguin Random House

Seo-Zindy, R., \& Heeks, R. (2017). Researching the Emergence of 3D Printing, Makerspaces, Hackerspaces and Fab Labs in the Global South: A scoping review and research agenda on digital innovation and fabrication networks. Electronic Journal of Information Systems in Developing Countries, 80(5), pp. 124. Retrieved from https://doi.org/10.1002/j.16814835.2017.tb00589.x

Senske, N. (2014). Digital Minds, Materials, and Ethics: Linking Computational Thinking and Digital Craft. In Rethinking Comprehensive Design: Speculative Counterculture, 19th CAADRIA Conference, Hong Kong. Retrieved from http://papers.cumincad.org/data/works/att/caadria2014_256.c ontent.pdf

Sperling, D. \& Herrera, P.C. (2015). Homo Faber. Digital Fabrication in Latin America. São Carlos, Brazil: Instituto de Arquitetura e Urbanismo de São Carlos

Sperling, D., Herrera, P.C. \& Scheeren, R. (2015). Migratory Movements of Homo Faber: Mapping Fab Labs in Latin America. In: Celani G., Sperling D., Franco J. (Eds) Computer-Aided Architectural Design Futures. The Next City - New Technologies and the Future of the Built Environment. CAAD Futures 2015. Communications in Computer and Information Science, vol 527. (pp. 405-421. Berlin: Springer. https://doi.org/10.1007/978-3-662-47386-3_22

Stokes, L. (2014). New Territories: Laboratories for Design, Craft and Art in Latin America. Madrid: Turner.

Treré, E. \& Barranquero, A. (2017). Tracing the Roots of Technopolitics: Towards a North-South Dialogue. In F. Sierra \& T. Gravante (Eds.), Networks, Movements and Technopolitics in Latin America. Critical Analysis and Current Challenges (pp. 43-63). Cham, Switzerland: Palgrave Macmillan.

Troxler, P. (2010). Commons-based Peer-Production of Physical Goods Is there Room for a Hybrid Innovation Ecology? Paper presented at the 3rd Free Culture Research Conference, Berlin, October 8-9.

Troxler, P. \& Wolf, P. (2010). Bending the Rules. The Fab Lab Innovation Ecology. Paper presented at the $11^{\text {th }}$ International CINet Conference, Zurich, September 5-7.

Valsiner, J., Lutsenko, A \& Antoniouk, A. (2018). Sustainable Futures for Higher Education. The Making of Knowledge Makers. Cham:Springer.

Velásquez, M. (2018). Jóvenes rurales e inclusión digital en Bolivia: el proyecto Saraña en Ancoraimes. In C. Cobo et al. (Eds.), Jóvenes, transformación digital y formas de inclusión en América Latina. (pp. 412-424). Montevideo: Penguin Random House 
Waldman-Brown A., Obeng GY., Adu-Gyamfi Y., Langevin S., \& Adam A. (2013) Fabbing for Africa's Informal Sector. Paper presentet at the 9th International Fab Lab Conference, FAB9, Yokohama, Japan, 21-27 August 2013.
Walter-Herrmann, J. (2013). FabLabs - A Global Social Movement. In J. Walter-Herrman \& C. Büching (Eds.), FabLabs of Machines, Makers and Inventors (pp. 33-45). Wetzlar: Majuskel Medienproduktion $\mathrm{GmbH}$. 